\title{
The Profiles of Stevens-Johnson Syndrome (SJS) and Toxic Epidermal Necrolysis (TEN) Patients in Tertiary Hospital
}

\author{
William Andrew Isaac ${ }^{1}$, Damayanti², Nurmawati Fatimah ${ }^{3}$, Afif Nurul Hidayati ${ }^{2,4}$ \\ ${ }^{1}$ Undergraduate of Medicine Program, Faculty of Medicine, Universitas Airlangga, Surabaya, \\ Indonesia \\ ${ }^{2}$ Department of Dermatology and Venereology, Faculty of Medicine, Universitas Airlangga/Dr. \\ Soetomo General Academic Hospital, Surabaya, Indonesia \\ ${ }^{3}$ Department of Pharmacology, Faculty of Medicine, Universitas Airlangga, Surabaya, Indonesia \\ ${ }^{4}$ Universitas Airlangga Teaching Hospital, Surabaya, Indonesia
}

\begin{abstract}
Background: Stevens-Johnson syndrome (SJS) and toxic epidermal necrolysis (TEN) are severe eruptive skin reactions that can cause death. The incidence of SJS and TEN cases in the United States is 1.5-9.6 per 1,000,000 per year. Drugs are the primary etiology of SJS and TEN. Some drugs are at high risk and used frequently. The SJS and TEN mortality rates were relatively high, with SJS 4.8\%, SJS / TEN overlap 19.8\%, and TEN 14.8\%. In Indonesia, there are lack of studies on the SJS and TEN. This study is needed to determine the epidemiological profile of SJS and TEN. Purpose: This study aimed to describe SJS and TEN patients' profiles. Methods: Drug-induced SJS and TEN cases from January 2016 to December 2019 were evaluated from the medical records patients' profile, incidence, suspected drugs, risk factors, and comorbidities of SJS and TEN were described. Result: There were 28 SJS and TEN patients, comprising of 24 SJS patients (85.7\%), 3 TEN patients $(10.7 \%)$, and 1 SJS overlapping TEN patients (3.5\%). The most common suspected drugs were paracetamol $(22.2 \%)$, carbamazepine (20.4\%), cefadroxil (8.8\%), and ciprofloxacin $(8.8 \%)$. Women $(53.5 \%)$ experienced more severe drug eruptions than men (46.4\%). The largest age group was 25-44 years (35.7\%). Most comorbidities were epilepsy $(21 \%)$, diabetes (15.7\%), hypertension (15.7\%), and stroke (15.7\%). Conclusion: The most common manifestation was SJS with paracetamol as the most common suspected drug, followed by carbamazepine.
\end{abstract}

Keywords: Stevens-Johnson syndrome, toxic epidermal necrolysis, skin eruption, mortality.

Correspondence: Damayanti, Department of Dermatology and Venereology, Faculty of Medicine, Universitas Airlangga, Dr. Soetomo General Academic Hospital, Jl. Mayjen Prof. Dr. Moestopo No. 6-8 Surabaya 60131, Indonesia. Phone: +62315501609, e-mail: dr_damayanti_bs@yahoo.com.

\section{BACKGROUND}

Toxic epidermal necrolysis (TEN) and StevensJohnson syndrome (SJS) are acute life-threatening mucocutaneous reactions. Such reaction is characterized by erythematous eruptions of the skin and extensive shedding of the epidermis and the mucous membranes' erosion. The severity of SJS / TEN is indicated by the degree of epidermal ablation. In SJS, epidermal ablation affects less than $10 \%$ of the body surface, whereas, in TEN, epidermal ablation affects more than $30 \%$ of the body surface. If $10 \%$ $30 \%$ of the body surface is affected, this is called an overlapping SJS / TEN. ${ }^{1}$

Stevens-Johnson syndrome and toxic epidermal necrolysis are rare but life-threatening severe cutaneous adverse reactions (SCAR). Overall, SJS and TEN incidences are estimated to be 1-6 cases per million people per year, and $0.4-1.2$ cases per million per year. ${ }^{1}$ The incidence of SJS and TEN cases in the United States is 1.5-9.6 per 1,000,000 population per year. ${ }^{2}$ In France, SJS and TEN incidence and mortality are 6.5 and 0.9 per $1,000,000$ population per year. ${ }^{3}$ Meanwhile, in China, the incidence of SJS and TEN cases is $1.8 \%$ of the hospital population. In Singapore, $0.7 \%$ of cases of cutaneous adverse drug reactions (CADR) are SJS and TEN. SJS and TEN are the most severe drug eruptions. ${ }^{4}$ Previous study conducted in Dr. Soetomo General Academic Hospital Surabaya reported that adverse drug reaction (ADR) occurred in $6.5 \%$ of patients admitted to the hospital, with a mortality rate of $2 \% .{ }^{5}$ Globally, mortality rate for SJS and TEN are high. Therefore, a thorough analysis of epidemiological data is needed. Although some hospital-based SJS and TEN incidences have been reported in Indonesia, its epidemiological data representing the general population's incidence is still lacking. There is no systematic study to describe SJS and TEN nationally.

Drugs are the primary etiology of SJS and TEN. Another reported etiology of SJS and TEN is a skin infection, but it is rare. The EuroSCAR study evaluated drugs according to their degree of risk for causing SJS 
and TEN. The drugs with the highest risk are allopurinol, carbamazepine, cotrimoxazole, other sulfonamide antibiotics, sulfasalazine, lamotrigine, nevirapine, oxicam-type non-steroidal antiinflammatory drugs (NSAIDs), phenobarbital, and phenytoin. Moderate risk drugs include cephalosporins, macrolides, quinolones, tetracyclines, and acetic acid-type NSAIDs. In previous studies, lowrisk drugs were not associated with a measurable risk, including beta-blockers, angiotensin-converting enzyme inhibitors, calcium channel blockers, and sulfonamide-based thiazide diuretics, sulfonylurea anti-diabetes, insulin, and propionic acid derivatives NSAIDs. SJS and TEN epidemiological studies in Southeast Asia showed that the main causative drugs were carbamazepine (17\%), allopurinol (15\%), sulfonamide antibiotics (12\%), phenytoin (9\%), NSAIDs ( $8 \%$ ), lamotrigine (2\%), phenobarbital (1\%), and B-lactam antibiotics (13\%). ${ }^{6,7}$

The SJS and TEN mortality rates is high, the SJS mortality rate is $4.8 \%$, the SJS / TEN overlaps are $19.8 \%$, and the TEN cases are $14.8 \% .^{2}$ Due to the high SJS and TEN mortality rates, understanding and cautious drug use are needed because most of the drugs with a risk of SJS and TEN can be purchased without a prescription.

This study aimed to contribute towards the understanding and awareness of irrational drug use to prevent and reduce the morbidity and mortality of SJS and TEN.

\section{METHODS}

This was a descriptive study with a retrospective approach, aiming to evaluate SJS and TEN patients' profiles at Dermatology and Venereology Inpatient Installation Dr. Soetomo General Academic Hospital Surabaya. Secondary data were obtained from all medical records of patients with drug-induced SJS and TEN cases from January 2016 to December 2019. Medical records were evaluated based on patient profiles, number of cases, suspected drugs, risk factors, and comorbidities. This study has been reviewed by the
Ethics Committee at Dr. Soetomo General Academic Hospital Surabaya (No. 1562/KEPK/X/2019).

\section{RESULT}

In 2016-2019, 24 SJS patients $(85.7 \%), 3$ TEN patients $(10.7 \%)$, and 1 patient with overlapping SJS / TEN patient (3.5\%) were hospitalized at Dermatology and Venereology Inpatient Installation Dr. Soetomo General Academic Hospital Surabaya (Table 1).

This study found a total of 44 suspected drugs that cause severe eruptions in the form of SJS / TEN. The patient might be prescribed more than one suspected drug. Of all SJS and TEN cases, 11 (39.2\%) cases prescription were consisted of one suspected drug, and $14(50 \%)$ cases consisted of several suspected drugs. The other $3(10.7 \%)$ cases of unknown drug history were also found because the patients could not remember the medical record.

The most common suspected drug classes were NSAIDs in 14 patients (31.8\%), 13 patients with anticonvulsants $(29.5 \%), 11$ patients with antibiotics (25\%), and other drugs (Table 2). The other drug classes included antiemetics in 1 patient $(2.2 \%)$ and antihypertensive in 1 patient $(2.2 \%)$. Cephalosporins were the most common type of suspected drug (50\%) in antibiotics, followed by fluoroquinolones (41.6\%).

The most widely used drugs were paracetamol in 10 patients $(22.2 \%)$, carbamazepine in 9 patients $(20.4 \%)$, cefadroxil in 4 patients $(8.8 \%)$, and ciprofloxacin in 4 patients $(8.8 \%)$ (Table 3$)$.

Most SJS and TEN patients were female, with 15 female patients $(53.5 \%)$ and 13 male patients (46.4\%) (Picture 1). The age distribution was set as per WHO's recommendation. Based on table 4, the 25-44 years age group experienced SJS and TEN more often, with a total of $10(35.7 \%)$ cases. The patients' mean age was 38 years old, with a median value of 39.5 . The youngest drug eruption patient in this study was three years old, and the oldest patient was 75 years old.

In this study, only $1(3.5 \%)$ patients had a history of previous drug eruptions that was SJS and erythroderma, which later changed to TEN. In comparison, $27(96.5 \%)$ of patients had no history of previous drug eruptions.

Table 1. The number of SJS and TEN

\begin{tabular}{ll}
\hline & Number of patients \\
\hline SJS & 24 \\
TEN & 3 \\
SJS/TEN & 1 \\
\hline Total & 28 \\
\hline SJS = Steven-Johnson syndrome & \\
TEN = toxic epidermal necrolysis & \\
SJS/TEN = Steven-Johnson syndrome overlapping toxic epidermal necrolysis
\end{tabular}


Table 2. Distribution of suspected drug classes causing SJS and TEN

\begin{tabular}{ll}
\hline Suspected drug classes & Number of drugs \\
\hline NSAID & 14 \\
Anticonvulsant & 13 \\
Antibiotic & 11 \\
Other drug classes & 6 \\
\hline Total & 44 \\
\hline NSAID = non-steroidal anti-inflammatory drug, a patient may have a history of using more than one drug \\
SJS = Steven-Johnson syndrome
\end{tabular}

Table 3. Distribution of suspected drugs causing SJS and TEN

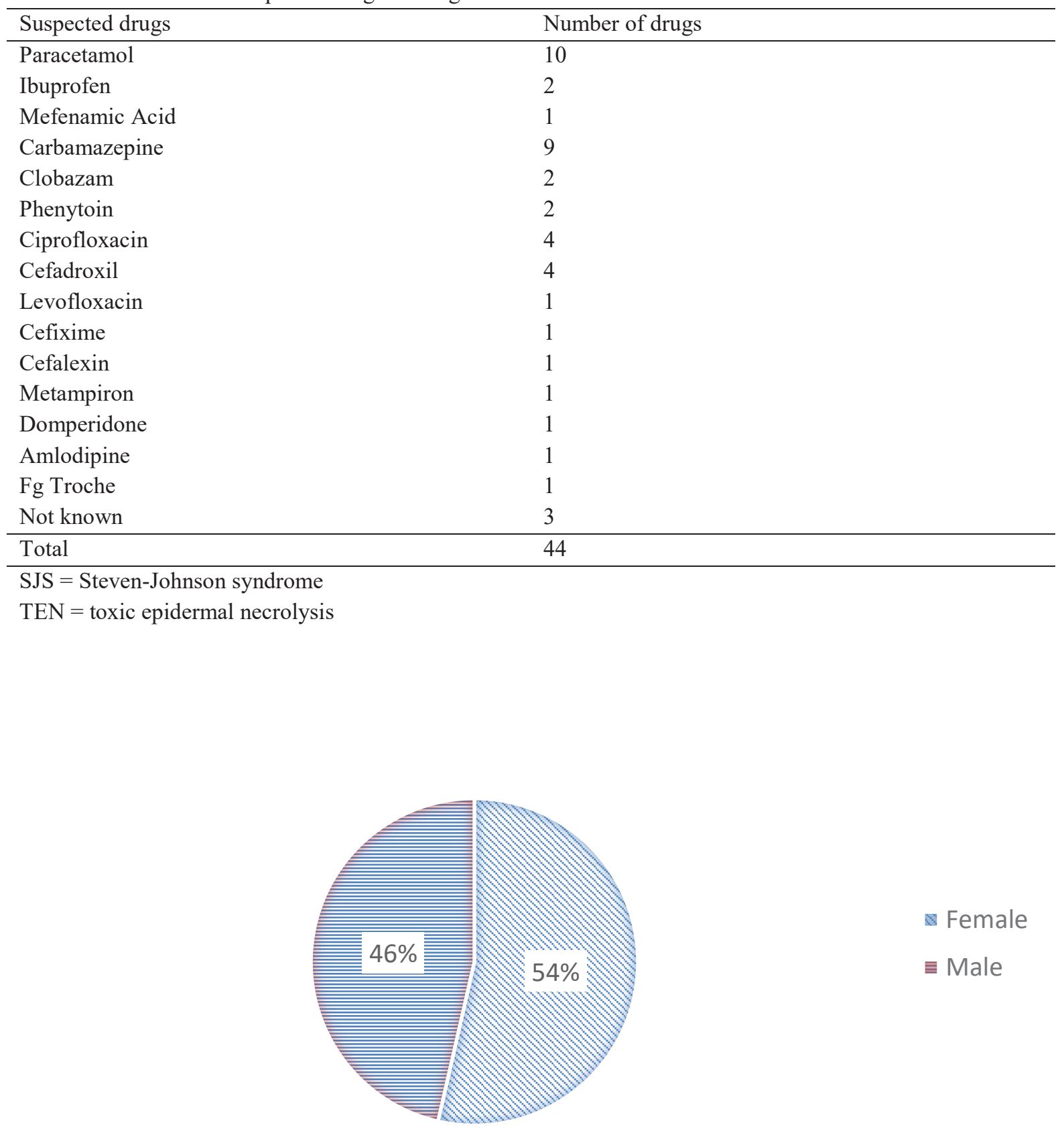

Figure 1. The sex distribution of Steven-Johnson syndrome (SJS) and toxic epidermal necrolysis (TEN) patients. 
Table 4. The age distribution of SJS and TEN patients

\begin{tabular}{ll}
\hline Age group (years old) & Number of patients \\
\hline $1-4$ & 2 \\
$5-14$ & 1 \\
$15-24$ & 5 \\
$25-44$ & 10 \\
$45-64$ & 7 \\
$>65$ & 3 \\
\hline Total & 28 \\
\hline
\end{tabular}

SJS = Steven-Johnson syndrome

$\mathrm{TEN}=$ toxic epidermal necrolysis

Table 5. Distribution of SJS and TEN patients' comorbidities

\begin{tabular}{ll}
\hline Comorbidities & Number of patients \\
\hline Hypertension & 3 \\
Diabetes Mellitus & 3 \\
Stroke & 3 \\
Epilepsy & 4 \\
Heart disease & 2 \\
Secondary infection & 2 \\
Kidney stone & 1 \\
Brain tumor & 1 \\
\hline Total & 19 \\
\hline
\end{tabular}

SJS $=$ Steven-Johnson syndrome

$\mathrm{TEN}=$ toxic epidermal necrolysis

Based on table 5, the most common comorbidities in SJS and TEN patients were epilepsy in 4 patients $(21 \%)$, diabetes in 3 patients $(15.7 \%)$, hypertension in 3 patients $(15.7 \%)$, and stroke in 3 patients $(15.7 \%)$. A patient can have more than one comorbidities.

\section{DISCUSSION}

This study involved 24 SJS patients, 3 TEN patients, and 1 SJS / TEN overlap patient. Another study also showed a similarity with the proportion of hospitalized cases of SJS and TEN patients. A study conducted by Sousa-Pinto et al. showed that out of 132 SJS and TEN patients, the highest incidence of severe drug eruptions was SJS with 73 patients (55\%), TEN with 41 patients (31\%), and SJS / TEN overlap with 18 patients (14\%). ${ }^{8}$ Another study conducted by Mokhtari et al. also showed that out of 282 drug eruption patients, SJS was the most clinical manifestation of severe drug eruption, which was around $32 \%$ of the total cases. ${ }^{9}$ However, Carrasquillo et al. reported different findings. They reported 17 (57\%) TEN cases, 3 (10\%) SJS cases, and $10(33 \%)$ overlapped cases. ${ }^{10}$

In this study, 44 suspected causative drugs for SJS and TEN were found. The most alleged drug class causing the eruption was NSAIDs, as observed in 14 patients $(31.8 \%)$, anticonvulsants as observed in 13 patients $(29.5 \%)$, and antibiotics as observed in 11 patients $(25 \%)$. This finding is in line with several studies. The most common causative drug classes of SJS and TEN cases in Southeast Asia are antibiotics, anticonvulsants, NSAIDs, and allopurinol. ${ }^{7}$ However, no anti-gout drug was suspected to be the cause of the eruption in this study.

The suspected causative drugs of SJS and TEN in this study were paracetamol $(22.2 \%)$, carbamazepine (20.4\%), cefadroxil (8.8\%), and ciprofloxacin (8.8\%). Research in Korea showed that carbamazepine had caused the most severe drug eruption reactions. Of the total 56 SJS cases, $26(50 \%)$ of them were caused by carbamazepine, followed by lamotrigine $(21.4 \%) .{ }^{11}$ However, a study conducted in Vancouver General Hospital involving 64 patients with SJS and TEN reported that allopurinol was most likely to cause eruptions, contributing up to $20 \%$ of the total cases. ${ }^{12}$

In this study, paracetamol was the most common NSAID drug suspected to cause SJS and TEN, as observed in 10 patients $(22.2 \%)$. This result is in line with a previous study, reporting that paracetamol was the most suspected drug to cause eruptions, as observed in 7 patients. ${ }^{13}$ In comparison, seven other cases were patients with a history of taking more than one drug. Paracetamol is widely used in conjunction with cefadroxil $(n=3)$, carbamazepine $(n=2)$, cefixime ( $=1)$, ciprofloxacin $(n=1)$, domperidone $(n=1)$, and 
ibuprofen $(n=1)$. Other studies have reported that the incidence of SJS and TEN caused by paracetamol is quite rare. This shows that drugs that are considered potentially safe can cause severe hypersensitivity reactions in the form of SJS and TEN. Although a casecontrol study conducted in France found no significant risk associated with paracetamol use as a cause of SJS and TEN. ${ }^{14}$

Carbamazepine was the most common suspected anticonvulsant drug to cause SJS / TEN ( $n=9,20.4 \%)$. The incidence of SJS and TEN in patients with drug eruptions increases with risk factors in the form of specific alleles in the patient's human leukocyte antigen (HLA) for certain drugs. Genetic polymorphisms can determine changes in gene expression that affect the immune response, such as the HLA gene, which forms a specific type of immunological system-mediated drug eruption. ${ }^{15}$ Many studies suggest that carbamazepine is primarily responsible for SJS and TEN cases in the anticonvulsant class. A strong association was found between HLA-B*15:02 and SJS and TEN caused by carbamazepine. This was further validated from various studies in other populations in Asia. Studies in Vietnam show that SJS and TEN caused by carbamazepine are closely related to HLA$B * 15: 02$, increasing the risk of a severe drug eruption reaction. ${ }^{16} \mathrm{~A}$ recent study in Indonesia found that the HLA-B*15:02 allele in SJS and TEN caused by carbamazepine ( $\mathrm{n}=8 / 12,66.7 \%)$ was significantly more than in the carbamazepine-tolerant control group $(\mathrm{n}=4 / 17,29.4 \%)$ and also with normal controls $(\mathrm{n}=$ 54/236, 22.9\%). This indicates a strong relationship between the HLA-B*15:02 allele and the risk of SJS and TEN caused by carbamazepine in Java and Sunda. ${ }^{17}$ Therefore, HLA plays a crucial role in the risk factor of drug eruption, especially SJS and TEN.

The antibiotic classes that were most likely to cause SJS and TEN in this study were cephalosporin (n $=6)$ and quinolone $(n=4)$ with cefadroxil $(n=4,8.8 \%)$ and ciprofloxacin $(n=4.8,8 \%)$ at most. The results of this study are quite different from other studies. Research on antibiotics with severe drug eruptions conducted in Taiwan found that penicillin $(n=20$, $27 \%)$ and cephalosporins $(n=17,22.9 \%)$ were the classes that have caused the most eruptions, followed by quinolones $(n=5,6.7 \%) .{ }^{18}$ Meanwhile, research in Nigeria in 2004-2017 found that cotrimoxazole ( $\mathrm{n}=$ $143,33.5 \%)$ as an antibiotic often causes SJS and TEN. ${ }^{19}$ Immunopathology in allergic reactions to antibiotics lies in the formation of the hapten. According to that note, cephalosporins' stability and speed to form hapten are inferior to penicillin because of the dihydrothiazine ring. Penicillins' superiority in forming the hapten results in a higher risk of allergic reactions than cephalosporins. However, in this study, there was no patient with a history of penicillin use. This can be caused by avoiding penicillin allergy by switching to cephalosporins. Cephalosporins are broad-spectrum antibiotics. They are more often used for common infectious diseases such as upper respiratory infection. Cotrimoxazole is limited to certain diseases, for example, in the prophylaxis of HIV/AIDS, toxoplasmosis, or pneumonia. Also, cotrimoxazole has dangerous side effects and a risk of toxicity. Of several antibiotics classes, beta-lactams (penicillin and cephalosporins), cotrimoxazole, and quinolones are frequent antibiotic classes that cause hypersensitivity reactions. ${ }^{20}$

This study's largest age group was 25-44 years old, with $10(35.7 \%)$ patients. The youngest SJS and TEN patients were 3 years old, and the oldest patients were 75 years old. The mean age in the study was 38 years old. These results are quite similar to other studies. Previous research conducted at the Dr. Soetomo General Academic Hospital found that the largest age group for SJS and TEN patients were 2544 years old $(\mathrm{n}=18,48.6 \%)$. Research in China reported that the largest age group was $31-40$ years old $(\mathrm{n}=20,22.7 \%)$, and the mean age of SJS and TEN patients was $45 \pm 18$ years old. ${ }^{21}$

Physiological changes with age also affect the pharmacokinetics and pharmacodynamics of drugs. There is a reduction in muscle mass and water proportion in the body and increased total fat content at a later age. This affects the distribution of drugs that can increase toxicity, so it is necessary to adjust the dose according to the patient's age. The hepatic mass and perfusion will decrease by about $40 \%$ as the patient age, affecting drug metabolism and excretion. Glomerular filtration rate (GFR) will also decrease with age due to decreased renal mass, perfusion, and nephron function, thereby affecting drug elimination through the kidneys. Also, older patients are more sensitive to treatment effects than younger ones due to drug pharmacodynamics changes. Older patients are the most drug-consuming age group in developing countries. However, in this study, SJS and TEN incidences decreased at 45 years and older. This can be attributed to the decreasing intensity of drug use with age and older patients' unwillingness to seek treatment in health facilities. Knowledge, costs, and attitudes of people at a later age affect how they buy and consume drugs. In general, people of reproductive age take medication more often because they could not keep up with their productivity if they are sick. ${ }^{22}$

This study's largest gender was women, with 15 $(53.5 \%)$ patients, while men were $13(46.5 \%)$ patients. This is in line with the literature stating that SJS and 
TEN are more common in women with a sex ratio of 0.6 . However, a study conducted by Barvaliya et al. had male preponderance with 17 male patients and 15 female patients. ${ }^{1,23}$

Women experience drug eruption reactions more frequently. The risk difference between women and men in the immune response to certain drugs' consumption is not known with certainty. However, there are some differences between men and women in pharmacodynamics and pharmacokinetics. The difference in body size between men and women results in a greater drug distribution volume and faster clearance in men than women. Women have higher total body fat than men. This will increase the distribution of lipophilic drugs to women, for example, the benzodiazepine anticonvulsant drugs. Also, bioavailability in oral drug use increases in women, especially for CYP3A substrates. Other influencing factors are also present in conjugation, protein binding, and drug metabolism. Drug eruptions, especially on the skin, have an immunological etiology. Although the exact mechanism is unknown, sex differences in $\mathrm{T}$ cell activation and proliferation have increased drug eruptions in women. The same can be attributed to the higher prevalence of systemic lupus erythematosus and photosensitivity in women. ${ }^{24}$

The results of the history of comorbidities in this study did not differ significantly between them. The most common comorbidities in SJS and TEN patients were epilepsy $(n=4,15.3 \%)$, hypertension $(n=3$, $11.5 \%)$, stroke $(\mathrm{n}=3,11.5 \%)$, and diabetes $(\mathrm{n}=3$, $11.5 \%$ ). The results of a study in Portugal reported that the most common comorbidities in SJS / TEN patients were hypertension $(n=46,34.8 \%)$, diabetes $(n=22$, $16.7 \%)$, and chronic kidney disease $(n=15,11,4 \%){ }^{8}$ The Puerto Rican study also reported similarities with a history of comorbidities such as hypertension (33.3\%), asthma (23.3\%), diabetes (13.3\%), and epilepsy (10\%). ${ }^{10}$ In America, investigative literature stated that SJS was associated with malignancy, infection, HIV / AIDS, and other disorders such as epilepsy, systemic lupus erythematous, and kidney disease. ${ }^{2}$ Generally, patients who have a history of comorbid epilepsy potentially take more than one type of drug, increasing the risk of severe drug eruption reactions due to drug interactions, such as valproate acid and lamotrigine. Diabetes mellitus has been associated with poor hospitalization outcomes because patients with diabetes mellitus develop immunocompromised complications. Patients with diabetes mellitus have a higher complication rate with SJS and TEN than those without a history of diabetes mellitus. However, independent diabetes mellitus did not increase the incidence of complications. Also, SJS and TEN patients with diabetes mellitus would have a longer stay. ${ }^{25}$ Concomitant diseases in SJS and TEN patients affect the severity of the disease and the prognosis. SJS and TEN complications that should be monitored are sepsis, shock, fluid balance disorders, and electrolyte balance disorders.

In this study, SJS was the most common manifestation $(85.7 \%)$. NSAID was the most common offending drug classes $(31.8 \%)$ in the SJS and TEN cases, with paracetamol as the most drug suspected (22.2\%) followed by carbamazepine (29.4\%). Understanding and awareness can avoid irrational drug use, as common drugs could result in severe skin eruption. Also, educating patients can further prevent adverse drug reactions.

\section{REFERENCES}

1. Mockenhaupt M, Roujeau JC. Epidermal necrolysis (Stevens-Johnson syndrome and toxic epidermal necrolysis). In: Kang S, Amagai M, Bruckner A, Enk AH, Margolis DJ, McMichael AJ, et al., editors. Fitzpatrick's Dermatology. New York: McGraw-Hill Education; 2019.p.73345.

2. Hsu DY, Brieva J, Silverberg NB, Silverberg JI. Morbidity and mortality of Stevens-Johnson Syndrome and toxic epidermal necrolysis in United States adults. J Invest Dermatol 2016;136(7):1387-97.

3. Chaby G, Maldini C, Haddad C, Lebrun-Vignes $\mathrm{B}$, Hemery F, Ingen-Housz-Oro S, et al. Incidence of and mortality from epidermal necrolysis (Stevens-Johnson syndrome/toxic epidermal necrolysis) in France during 2003-16: a foursource capture-recapture estimate. Br J Dermatol 2020;182(3):618-24.

4. Wong SX, Tham MY, Goh CL, Cheong HH, Chan SY. Spontaneous cutaneous adverse drug reaction reports - An analysis of a 10-year dataset in Singapore. Pharmacol Res Perspect 2019;7(2):e00469.

5. Damayanti D, Umborowati MA, Anggraeni S, Prakoeswa CR, Hutomo M, Sukanto H. Clinicoepidemiological profile of severe cutaneous adverse drug reaction: A retrospective study. Berkala Ilmu Kesehatan Kulit dan Kelamin. 2019;31(1):1-6.

6. Mockenhaupt M, Viboud C, Dunant A, Naldi L, Halevy S, Bavinck JN, Sidoroff A, et al. StevensJohnson syndrome and toxic epidermal necrolysis: Assessment of medication risks with emphasis on recently marketed drugs. The EuroSCAR-study. J Invest Dermatol 2008;128(1):35-44. 
7. Lee HY, Martanto W, Thirumoorthy T. Epidemiology of Stevens-Johnson syndrome and toxic epidermal necrolysis in Southeast Asia. Dermatol Sin 2013;4(31):217-20.

8. Sousa-Pinto B, Araújo L, Freitas A, Correia O, Delgado L. Stevens-Johnson syndrome/toxic epidermal necrolysis and erythema multiforme drug-related hospitalisations in a national administrative database. Clin transl allergy. 2018;8(1):1-0.

9. Mokhtari F, Nikyar Z, Naeini BA, Esfahani AA, Rahmani S. Adverse cutaneous drug reactions: Eight year assessment in hospitalized patients. J Res Med Sci 2014;19(8):720.

10. Carrasquillo OY, Santiago-Vazquez M, Cardona R, Cruz-Manzano M, Figueroa LD. StevensJohnson syndrome and toxic epidermal necrolysis: a retrospective descriptive study. Int J Dermatol 2019;58(11):1293-9.

11. Park CS, Kang DY, Kang MG, Kim S, Ye YM, $\mathrm{Kim} \mathrm{SH}$, et al. Severe cutaneous adverse reactions to antiepileptic drugs: A nationwide registrybased study in Korea. Allergy Asthma Immunol Res 2019;11(5):709.

12. Miliszewski MA, Kirchhof MG, Sikora S, Papp A, Dutz JP. Stevens-Johnson syndrome and toxic epidermal necrolysis: An analysis of triggers and implications for improving prevention. Am J Med 2016;129(11):1221-5.

13. Damayanti D, Anggraeni S, Prakoeswa CRS, Hutomo M, Sukanto H. Epidemiologic study: severe cutaneous adverse drug reaction. Berkala Ilmu Kesehatan Kulit dan Kelamin 2017;29(2):151-7.

14. Lebrun-Vignes B, Guy C, Jean-Pastor MJ, GrasChampel V, Zenut M, French Network of Regional Centres of Pharmacovigilance and the French Investigators for Adverse Skin Reactions to Drugs. Is acetaminophen associated with a risk of Stevens-Johnson syndrome and toxic epidermal necrolysis? Analysis of the French Pharmacovigilance Database. $\mathrm{Br} \mathrm{J}$ Clin Pharmacol 2018;84(2):331-8.

15. Damayanti D. Konsep Patomekanisme Obat Terkini. Berkala Ilmu Kesehatan Kulit dan Kelamin. 2019;31(3):136-41.

16. Nguyen D van, Chu HC, Nguyen D van, Phan $\mathrm{MH}$, Craig T, Baumgart K, et al. HLA-B*1502 and carbamazepine-induced severe cutaneous adverse drug reactions in Vietnamese. Asia Pac Allergy 2015;5(2):68-77.

17. Yuliwulandari R, Kristin E, Prayuni K, Sachrowardi Q, Suyatna FD, Menaldi SL, et al. Association of the HLA-B alleles with carbamazepine-induced Stevens-Johnson syndrome/toxic epidermal necrolysis in the Javanese and Sundanese population of Indonesia: the important role of the HLA-B75 serotype. Pharmacogenomics 2017;18(18):1643-8.

18. Lin YF, Yang CH, Sindy H, Lin JY, Hui CYR, Tsai YC, et al. Severe cutaneous adverse reactions related to systemic antibiotics. Clin Infect Dis 2014;58(10):1377-85.

19. Oshikoya KA, Ogunyinka IA, Ogar CK, Abiola A, Ibrahim A, Oreagba IA. Severe cutaneous adverse drug reactions manifesting as StevensJohnson syndrome and toxic epidermal necrolysis reported to the national pharmacovigilance center in Nigeria: a database review from 2004 to 2017. Ther Adv Drug Saf 2020;11:1-18.

20. Khan DA, Banerji A, Bernstein JA, Bilgicer B, Blumenthal K, Castells M, et al. Cephalosporin allergy: current understanding and future challenges. J Allergy Clin Immunol 2019;7(7):2105-14.

21. Wang L, Mei XL. Retrospective analysis of Stevens-Johnson syndrome and toxic epidermal necrolysis in 88 Chinese patients. Chin Med J 2017;130(9):1062.

22. Lavan AH, Gallagher P. Predicting risk of adverse drug reactions in older adults. Ther Adv Drug Saf 2016;7(1):11-22.

23. Barvaliya M, Sanmukhani J, Patel T, Paliwal N, Shah H, Tripathi C. Drug-induced StevensJohnson syndrome (SJS), toxic epidermal necrolysis (TEN), and SJS-TEN overlap: A multicentric retrospective study. J Postgrad Med 2011;57(2):115.

24. Schwartz JB. The influence of sex on pharmacokinetics. Clin Pharmacokinet 2003;42(2):107-21.

25. Patel S, Shah R, Behbahani, S, Lambert WC. Effect of diabetes mellitus on hospital complications of Stevens-Johnson syndrome and toxic epidermal necrolysis patients. J Am Acad Dermatol 2019;81(4):196. 